\title{
Effect of Stem Cells Injection in Adult Male Albino Rats with Streptozotocin-Induced Diabetic Nephropathy
}

\author{
Kumlachew D. Dissassa', Mona H. Ali', Mona A. Hussain ${ }^{2 *}$, Alaa El-Din S. Abd \\ Al-Hamid ${ }^{3}$
}

Departments of ${ }^{1}$ Anatomy, ${ }^{2}$ Physiology, ${ }^{3}$ Clinical Pathology, Faculty of Medicine, Suez Canal University, Egypt

\begin{abstract}
Background: Diabetic Nephropathy is a leading cause of end-stage renal disease and the most frequent cause of mortality in patients with diabetes. Therefore, it is extremely important to identify novel interventions to halt the progression of diabetic nephropathy. Aim: This study investigated the effect of human umbilical cord blood (HUCB) derived $\left(\mathrm{CD}_{34} 4^{+}\right)$stem cells therapy on renal morphologic and functional changes and alterations of kidney enzyme levels in streptozotocin (STZ)-induced diabetic nephropathy in rats. Materials and Methods: Sixty adult male albino rats were included in this study and were randomly and equally divided into three groups. Group 'A' was the control, group 'B' was treated with STZ $(65 \mathrm{mg} / \mathrm{kg}$, IP) only and group ' $\mathrm{C}$ ' was treated with STZ and HUCB CD $34^{+}$stem cells $\left(1 \times 10^{6}\right.$ cells/ rat, IV). Results: Intravenous injection of CD $34^{+}$ cells caused significant improvement in blood glucose level $(438.3 \pm 67 \mathrm{mg} / \mathrm{dl}$ of group B vs. $141.8 \pm 23.4 \mathrm{mg} / \mathrm{dl}$ of group $C, p<0.01)$. Serum urea and creatinine levels were significantly lower of group $C$ than group B. The levels of alanine aminotransferase (ALT) and alkaline phosphatase (ALP) enzymes in the kidney tissues were significantly lower of group $C$ than group B. Histopathology of the kidney showed much better recovery of group $C$ than group B. Conclusion: HUCB $\mathrm{CD} 34^{+}$cells transplantation in diabetic rats is able to improve blood glucose level, partially ameliorate the renal damage in diabetic rats and consequently improve renal function, renal histology and renal enzyme levels alteration.
\end{abstract}

Keywords: diabetes, human umbilical cord blood, renal enzymatic alteration

\section{Introduction}

Diabetes mellitus (DM) has reached a worldwide growing epidemic diffusion. DM is associated with a significantly-reduced quality of life and represents an important health and social problem. Most importantly, DM leads to severe complications in many organs and tissues through the induction of microangiopathy and macroangiopathy ${ }^{(1)}$. According to the World Health Organization, the total number of people with DM is predictable to rise from
285 million in 2010 to 439 million in $2030^{(2)}$. In humans, diabetes mellitus is one of the most prevalent conditions with spontaneous manifestations. In animals, it can be induced by partial pancreatectomy or by the administration of diabetogenic drugs such as alloxan, streptozotocin, ditizona and anti-insulin serum ${ }^{(3)}$. Available treatments in diabetes mellitus do not guarantee a tight glycemic control, since patients do not often adhere well to the medical instructions. Thus, even under treatment, patients develop diabetic disease complica- 
tions including stroke, neuropathy, retinopathy and nephropathy ${ }^{(2)}$. Diabetic nephropathy (DN) is one of the devastating complications in patients with diabetes. It has been considered that about $25 \%-40 \%$ of patients with type 1 or type 2 diabetes develop nephropathy within 20-25 years of the onset of diabetes ${ }^{(4,5)}$. Studies have shown an association between specific diabetic complications and liver enzyme alterations, but only limited data are available on the possible association between diabetic nephropathy and kidney enzyme alterations $^{(6)}$. The current management of patients with $\mathrm{DN}$ is palliative therapeutic strategies includes the use of drugs to control hyperglycemia, blood pressure and proteinuria. In advanced stages, patients receive renal replacement therapy, which consists of hemodialysis and, if possible, kidney transplantation. Unfortunately, the latter is only useful when the kidney is cotransplanted with pancreatic beta-islets; if this is not done, renal failure reappears. Therefore, the need for therapeutic strategies to prevent, delay or revert $\mathrm{DN}$ is compelling $^{(2)}$. Cell therapies aim at the differentiation of stem cells into the specific cell type required to repair damaged or destroyed cells or tissues. Over recent years, cell therapy has been introduced in a variety of application areas, including cardiovascular repair, diabetes, musculoskeletal disorders and renal repair $^{(7)}$. Theoretically, cell-based therapies may have the advantage of acting through multiple mechanisms in disorders with highly complex pathophysiology, such as diabetic nephropathy, while pharmacological interventions often target only a single aspect of a disease ${ }^{(2,4)}$. Stem cells can be obtained from many sources. Human umbilical cord blood (HUCB) has many advantages as a source of stem cells and it was proved that HUCB can be collected safely for both, mother and child, after delivery ${ }^{(8)}$. It has advantages of easy procurement, low risk of transmitting infections, immediate availability, and immune tolerance, allowing successful transplantation despite HLA disparity and it could be cryopreserved. Moreover, cord blood can be obtained noninvasively and frequently in contrast to invasive bone marrow aspiration ${ }^{(9)}$. So this study investigated the effect of HUCB derived $\left(\mathrm{CD}_{34} 4^{+}\right)$stem cell therapy on renal morphological and functional changes and alterations of kidney [enzymes] levels in STZ-induced diabetic nephropathy in rats.

\section{Materials and Methods}

This study was performed at the Anatomy, Physiology and Gynecology \& Obstetric Departments, Faculty of Medicine, Suez Canal University, Ismailia, Egypt after being approved by the ethical committee. HUCB samples were collected in Gynecology \& Obstetric Department. Stem cell separation and animal housing were done in Physiology Department. Histopathological studies were done in Anatomy Department.

\section{Animals}

Sixty adult male albino Sprague-Dawly rats (weight 150-200 gm) purchased from center for experimental animals, Faculty of Veterinarian Medicine, Zagazig University. All rats were left to acclimatize for one week prior to the experiment. Rats were housed in plastic cages at controlled room temperature (22-24 C) with 12 hour diurnal (day and night change) with free access to standard pellet animal diet and tap water. The animals were equally and randomly divided into three groups; A, B and C. Group $A$ was the control, group $B$ was treated with STZ (Sigma Chemical Co., Egypt) only, and group $C$ was treated with STZ+ HUCB CD $34^{+}$stem cells.

Induction of Diabetes Mellitus

After an overnight fast, the animals of 
groups $B$ and $C$ were injected with a single dose of STZ $(65 \mathrm{mg} / \mathrm{kg})$ intraperitoneally in sodium citrate buffer at $\mathrm{pH} 4 \cdot 5^{(10)}$. The animals were given a dextrose solution ( $5 \%$ ) [3 $\mathrm{ml} / \mathrm{rat}$, sic] to prevent hypoglycemia. Diabetes was confirmed when blood glucose level becomes $\geq 300 \mathrm{mg} / \mathrm{dl}$ within 72 hours after STZ treatment.

Collection and isolation of HUCB CD34+ cells Sterile collection tubes $(50 \mathrm{ml})$, containing $5 \mathrm{ml}$ citrate phosphate dextrose adenine-1 (CPDA-1) as an anticoagulant, were used for collection of the human umbilical cord blood. Mononuclear cells were isolated by Ficoll-Hypaque density gradient centrifugation (Sigma Chemical Co., Egypt). Separation of CD34 positive progenitor cells was carried out with immunomagnetic separation technique by Dynal $\mathrm{CD}_{3} 4^{+}$progenitor cell selection system $^{(11)}$.

\section{Transplantation of HUCB CD34+ cells}

$0.2 \mathrm{ml}$ of phosphate buffer saline solution was added to the $\mathrm{CD}_{34}{ }^{+}$cell pellet for injection in the tail vein in a dose of $1 \times 10^{6}$ cells/ rat during the second week after diabetes induction ${ }^{(11)}$. After diabetes induction, all groups were followed up with regular measurement of blood glucose level every week by glucometer (Medismart, Switzerland). Blood was obtained by snipping the rat tail under non fasting condition at 10 a.m. On sacrifice after 4 and 6 weeks postinduction of diabetes, animals were anesthetized and blood collection was done for serum creatinine and urea assays then both kidneys were removed for histopathology and [renal enzymes] assay.

\section{Serum creatinine and urea assay}

Serum levels of creatinine and urea were assayed by the Kinetic Jaffe method and kinetic UV method respectively, using Roche diagnostic kits on Hitachi autoanalyzer 912.
Histopathology of the kidneys and enzymatic estimation

The abdomen wall was opened and kidneys were identified and observed for any gross appearance and color change and tissues were preserved for histopathological studies and enzymatic estimation. For histopathology, the right kidney tissues were immediately transferred to $10 \%$ formalin saline for paraffin embedding and staining with Hematoxylin \& Eosin (H\&E) stain. For enzyme estimation, the left kidney tissues were immediately excised, washed in icecold saline, blotted on tissue paper and immediately transferred to phosphate buffer ( $\mathrm{pH}$ 7.4) for preservation. Kidney tissues were homogenized $\left(100 \mathrm{~g} \mathrm{~L}^{-1}\right)$ in cold (0.14 Mol L ${ }^{-1}$ ) KCl using a Teflon Homogenizer. Homogenates were centrifuged at temperature $4^{\circ} \mathrm{C}$ and 5000 RPM for 20 minutes, placed in cold chambers. Supernatants were stored at $-20^{\circ} \mathrm{C}$ until analysis ${ }^{(6)}$. Aspartate aminotransferase (AST), alanine aminotransferase (ALT) and alkaline phosphatase (ALP) were estimated with standard spectrophotometer techniques ${ }^{(12)}$.

\section{Statistical analysis}

Data are shown as means \pm SEM. The statistical significance of the changes in blood glucose, urea and creatinine levels and kidney enzyme levels of the study groups were evaluated by using the ONE-WAY ANOVA test of variance, Tukey's HSD post hoc test and T test. The difference was regarded as statistically significant at a $P$ value $<0.05$. Statistical analysis was done with the statistical package of social science (SPSS) computer software and data were presented in the form of tables and figures.

\section{Results}

HUCB $\mathrm{CD}_{34} 4^{+}$injection into streptozotocin induced diabetic nephropathy improved blood glucose levels in rats Approximately $1 \times 10^{6}$ HUCB CD34+ cells 
were injected intravenously into the diabetic rats of group $C$ during the second week after diabetes induction. The blood glucose levels of group $C$ (Figure 1 ) were significantly lower than group $B$ by the end of the third week after diabetes induction and this improvement became highly significant by the end of fifth week after diabetes induction and remained till the end of the study.

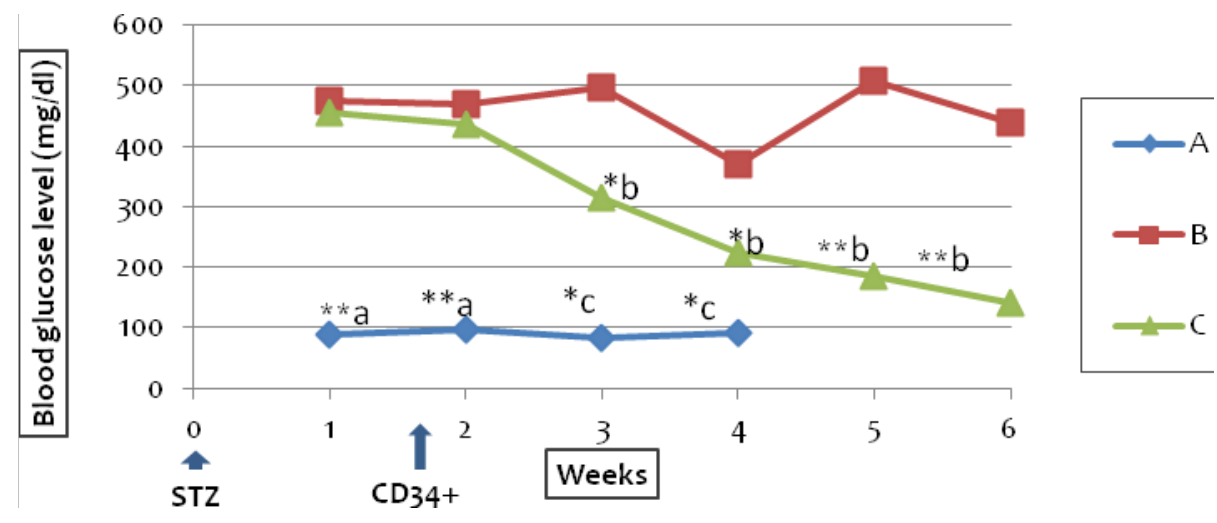

Figure 1: Comparison of blood glucose levels among the studied groups

A: Group A, normal control group; B: Group B, STZ only treated group; C: Group C, STZ + HUCB CD34+ stem cells treated group; a: significant difference between group $A$ and other study groups $B$ and $C$; b: significant difference between group B and group C; c: significant difference between group A and groups B

HUCB CD34+ injection into rats with streptozotocin-induced diabetic nephropathy improved blood urea, creatinine, renal ALT and ALP enzyme levels

Diabetic rats of group $B$ which did not receive stem cells showed a highly significant increase in serum urea levels $(p=0.005)$ and a significant increase in serum creatinine levels ( $p=0.035)$. Rats of group $C$ had normal serum urea and creatinine levels. The levels of ALT enzyme in renal tissues were significantly higher in diabetic rats of group $B$ than rats of group $C$ that received stem cells (246.8 \pm 52.7 vs. $96.3 \pm 9.7$ respectively, $p=0.04)$. The levels of AST enzyme in renal tissues showed no significant difference between group $B$ and group $C$ and were higher in diabetic rats of group $B$ and rats of group $C$ than normal control rats of group A $(409 \pm 32.9$ and $332.3 \pm 35.7$ vs. $108.5 \pm 7.5$ respectively $p=0.003)$. The levels of ALP enzyme in renal tissues were signifi- cantly higher in diabetic rats of group B than rats of group $C$ that received stem cells $(p=0.003)$. These results were obtained four weeks after diabetes induction (Table 1), and six weeks after diabetes induction (Table 2).

\section{Histopathological Results}

Sections of the kidney stained by H\&E of group A showed the normal malpighian renal corpuscles surrounded by proximal and distal convoluted tubules (Figure 2). The gross examination of the kidneys of group $B$ showed enlarged and edematous kidneys and darkness on the external surface. $\mathrm{H} \& \mathrm{E}$ stained sections, showed mild sub-capsular edema, vacuolar degeneration and cloudy swelling of some renal tubules and collecting ducts. Proliferation of the renal glomerular capillaries was seen. Some of the renal tubules exhibited eosinophilic material in their lumens; large number of mac 
rophages and lymphocyte infiltration were noticed within the renal tissue. Moreover, focal hemorrhages were noticed in the medullary area of the kidney (Figures 3\&4).

Table 1: Comparison of blood urea, creatinine levels, and kidney enzymes among the studied groups (4 weeks after induction of diabetes)

\begin{tabular}{|l|cc|cc|cc|c|}
\hline \multirow{2}{*}{ Variables } & \multicolumn{2}{|c|}{ Group A } & \multicolumn{2}{c|}{ Group B } & \multicolumn{2}{c|}{ Group C } & \multirow{2}{*}{ P-value } \\
\cline { 2 - 7 } & Mean & SEM & Mean & SEM & Mean & SEM & \\
\hline Serum urea (mg/dl) & 32.5 & 2.5 & $136.6^{\mathrm{a}}$ & 15 & 39.5 & 19.4 & 0.005 \\
Serum creatinine (mg/dl) & 0.47 & 0.11 & $0.9^{\mathrm{b}}$ & 0.04 & 0.46 & 0.14 & 0.035 \\
Kidney ALT (U/L) & 140.5 & 83.5 & $246.8^{\mathrm{b}}$ & 52.7 & 96.33 & 9.7 & 0.044 \\
Kidney AST (U/L) & $108.5^{\mathrm{c}}$ & 7.5 & 409 & 32.95 & 332.33 & 35.7 & 0.003 \\
Kidney ALP (IU/L) & 814.5 & 372.5 & $2650.8^{\mathrm{a}}$ & 282.1 & 1578.7 & 155.7 & 0.003 \\
\hline
\end{tabular}

Group A: normal control group; Group B: STZ only treated group, Group C: STZ + HUCB CD34+ stem cells treated group

${ }^{a}=$ significant difference between group $B$ and other study groups $A$ and $C$

${ }^{b}=$ significant difference between group $B$ and group $C$

${ }^{c}=$ significant difference between group $A$ and other study groups $B$ and $C$

Table 2: Comparison of blood urea and creatinine levels and kidney enzymes between groups $B$ and C (6 weeks after induction of diabetes)

\begin{tabular}{|l|cc|cc|c|}
\hline \multirow{2}{*}{ Variables } & \multicolumn{2}{|c|}{ Group B } & \multicolumn{2}{c|}{ Group C } & \multirow{2}{*}{ P-value } \\
\cline { 2 - 5 } & Mean & SEM & Mean & SEM & \\
\hline Serum urea (mg/dl) & 118 & 22.5 & 35 & 8.2 & 0.008 \\
Serum creatinine (mg/dl) & 1.51 & 0.4 & 0.43 & 0.02 & 0.031 \\
Kidney ALT (U/L) & 330.86 & 33.8 & 118.5 & 20.8 & $<0.001$ \\
Kidney AST (U/L) & 348 & 39 & 380.5 & 35 & 0.56 \\
Kidney ALP (IU/L) & 2253.3 & 234.1 & 1063.5 & 141.3 & 0.002 \\
\hline
\end{tabular}

Group A: normal control group; Group B: STZ only treated group, Group C: STZ + HUCB CD34+ stem cells treated group

${ }^{a}=$ significant difference between group $B$ and other study groups $A$ and $C$

b=significant difference between group $B$ and group $C$

${ }^{c}=$ significant difference between group $A$ and other study groups $B$ and $C$

Investigated kidney sections of diabetic rats of group B (six weeks post-induction) revealed severe histopathological alterations. Dilated glomeruli, an increase of hypertrophied mesangial cells within the glomerular tuft, expanded glomerular space, necrobiotic changes in the renal tubules and congested blood vessels were the most prominent features of the kidney (Figure 5). Some sections showed extended pyelonephritis and infiltration with macrophages (Figure 6). In the histological architecture of the kidney of group $C$ in comparison with those of diabetic animals of group $\mathrm{B}$, normal glomeruli, and normal renal tubules were encountered. In addition, the disappearance of the macrophages was noticed, but some of elliptical glomeruli with thickened capsule, invasion of endothelial cells and lymphocytic infiltration were noticed. Some kidney sections from animals in-group C showed sub-bowman's edema in few glomeruli and the others appeared normal. Some renal tubules showed mild vacuolar degeneration while the hemorrhages and the lymphocytic infiltrations were minimal. On the other hand, some of the renal tubules were suffered from cloudy swelling (Figure 7). After 6 weeks post induction, few sections of kidneys of group $C$ were nearly normal but showing chronic interstitial nephritis (Figure 8). 


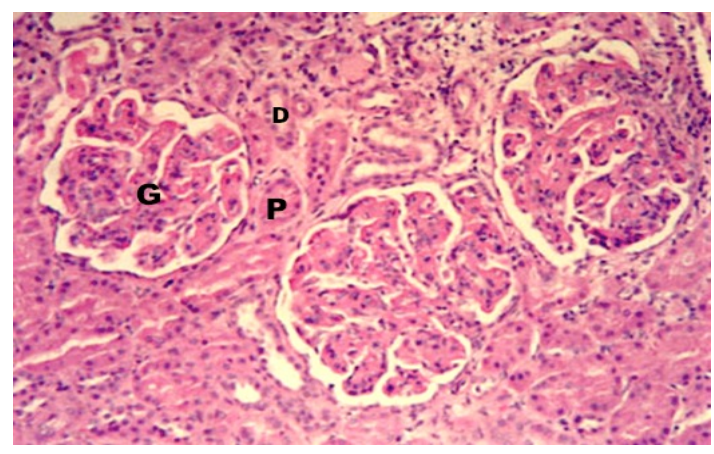

Figure 2: A photomicrograph of a section of the kidney of group A showing glomeruli (G), Proximal convoluted tubules $(P)$ and Distal convoluted tubules (D). H\&E x 400

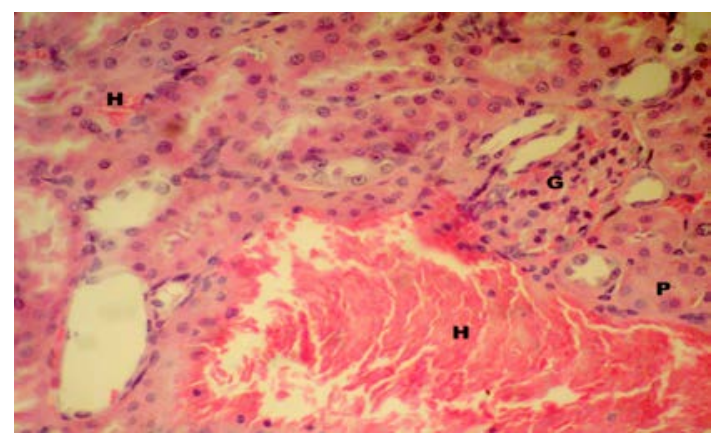

Figure 3: Photomicrograph of the section in the kidney of group B after 4 weeks showing hemorrhage $(H)$, cloudy swelling in the proximal tubule $(P)$, and proliferation of glomerular tuft (G). H\&E X 400

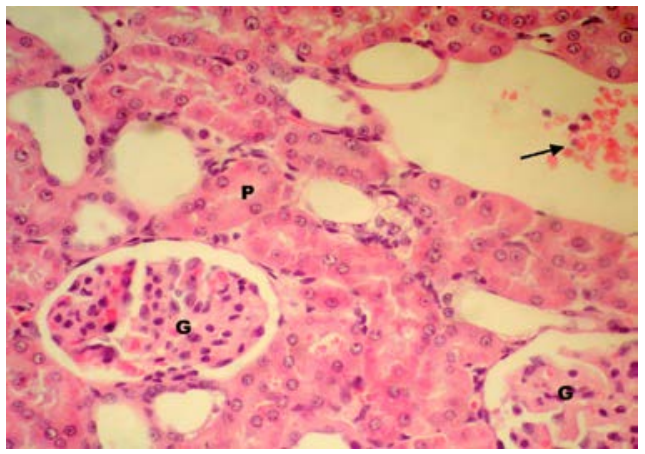

Figure 4: A Photomicrograph of a the section in the kidney of group B after 4 weeks showing congestion and proliferation of glomerular tuft (G), lymphocyte infiltration (arrow) and cloudy swelling of proximal tubules (P). $\mathrm{H} \& \mathrm{E} \times 400$

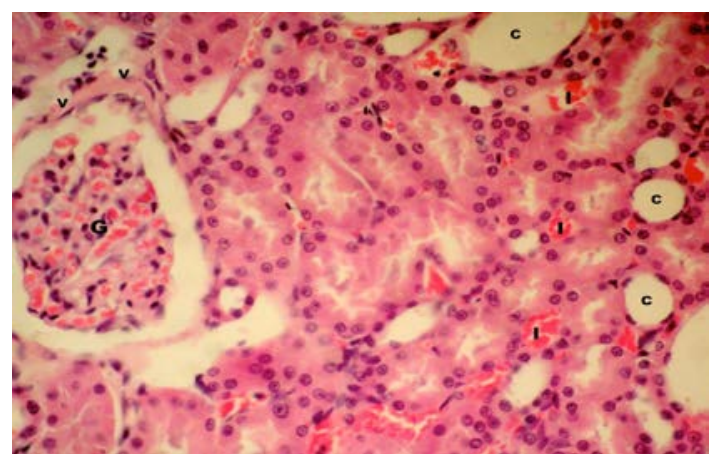

Figure 5: Photomicrograph of a section in the kidney of group B after 6 weeks showing congestion of glomerular tuft (G) massive cystic lumen $(C)$ and inter tubular capillary congestion (I). H\&E x 400

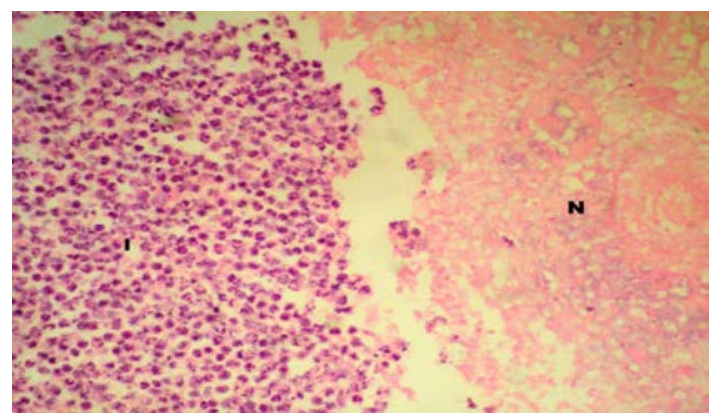

Figure 6: Photomicrograph of the section in the kidney of group B after 6 weeks showing inflammatory cells including pus cells (I), macrophages and necrotic renal tissue (N). $\mathrm{H} \& \mathrm{E} \times 400$

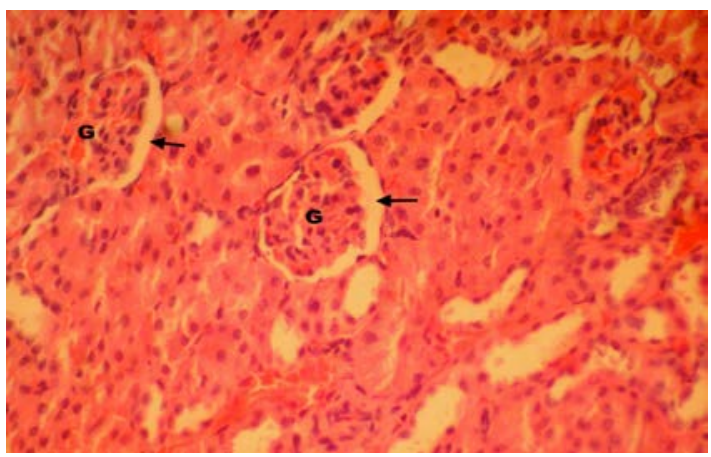

Figure 7: Photomicrograph of the section in the kidney of group $C$ after 4 weeks showing periglomerular and renal tubules suffering from cloudy edema arrows. H\&E X 400 


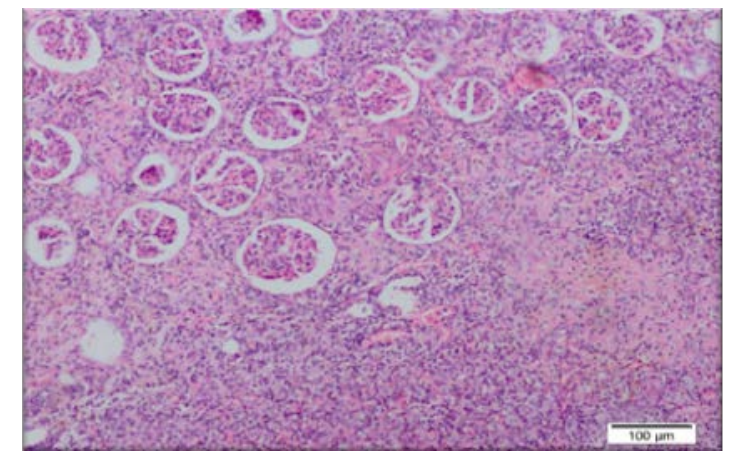

Figure 8: Photomicrograph of the section in the kidney of group C after 6 weeks, nearly normal, but showing chronic interstitial nephritis (contracted kidney). H\&E X 200

\section{Discussion}

The main observation made here is that transplantation of HUCB $\mathrm{CD} 34^{+}$stem cells partially ameliorates streptozotocin induced diabetic nephropathy in rats. The results of this study included partial improvement in structural changes in kidney of diabetic animals, normalization of both serum urea and creatinine levels and decreasing the alteration in ALT and ALP enzyme levels in kidney of diabetic animals that were transplanted by $\mathrm{HUCB} \mathrm{CD}_{34}{ }^{+}$. This observation is consistent with other studies, in which one of them, Lee et $\mathrm{al}^{(13)}$ reported that, transplantation of the multipotent stromal cells from human marrow improved glomerular morphology, decreased mesangial thickening, and decreased macrophage infiltration in kidney of diabetic animals ${ }^{(13)}$. In another study, HUCB mononuclear cells offered more corrective results in streptozotocin induced diabetic nephropathy than pioglitazone ${ }^{(9)}$. In the present study, normalization of blood glucose levels of group $C$ raises the possibility that improvement of renal structure and function in this group was secondary to the decrease in blood glucose level. This finding could be explained by the competence of $\mathrm{CD}_{34}{ }^{+}$stem cells to differ- entiate into insulin-producing beta cells $s^{(14)}$. Another explanation is that $\mathrm{CD}_{3} 4^{+}$stem cells stimulated recovery and endogenous regeneration of pancreatic islets ${ }^{(11,13)}$, followed by decrease of glycemia and glycosuria, factors important for damaging renal cells ${ }^{(14)}$. However, Wang et al., 2013 provided clear evidence that the injected mesenchymal stem cells 30 days after induction of diabetes in rats by streptozotocin did prevent the development of diabetic nephropathy with no improvement in blood glucose levels ${ }^{(15)}$. So, another mechanism(s) may be involved in diabetic nephropathy improvement after stem cell transplantation. "Development and progression of diabetic nephropathy are characterized by inflammatory infiltrates. The macrophage is a central mediator of renal vascular inflammation, and its accumulation is a feature of diabetic nephropathy. Macrophages mediate diabetic injury through a variety of mechanisms, including production of reactive oxygen species, cytokines, and proteases, which result in tissue damage. Components of the diabetic milieu, including high glucose, AGEs, and oxidized LDL, promote macrophage accumulation via induction of chemokines and adhesion molecules, and macrophage activation within diabetic kidneys"(16). This study shows that HUCB CD34 $4^{+}$transplantation decreased macrophage infiltration in the kidneys of diabetic animal of group $C$ leading to the improvement of the diabetic nephropathy. The interpretation of this data may be that the injected HUCB CD $34^{+}$ engrafted the diabetic kidneys, regulated the immune response ${ }^{(14)}$, and decreased macrophage infiltration resulting in the improvement of the diabetic nephropathy. Hasein et $\mathrm{al}^{(11)}$ in a study of the effect of HUCB CD $34^{+}$transplantation in diabetic mice reported that human insulin was detected in the pancreatic islets in $33 \%$ of 
treated mice, indicating that human umbilical cord blood $\mathrm{CD}_{34}+$ cells might change into insulin secreting cells in vivo and contribute to the improvement of hyperglycemia in diabetic mice ${ }^{(11)}$. Additionally, [Lee et al. ${ }^{(13)}$ in their previously mentioned study reported that, a small percentage of transplanted stem cell engrafted the diabetic kidney glomeruli and differentiated into endothelial cells ${ }^{(13)}$. Taken together, these data raise the possibility that transplanted stem cells engrafted the injured tissues and were able to differentiate into the cell type of the injured tissues. Stem cell transplantation may treat both the hyperglycemia and renal damage seen in diabetes and with systemic administration of stem cells in diabetes, many damaged tissues will be improved $^{(13)}$.

Another observation made here is that, transaminases (ALT and AST) and ALP enzyme levels significantly increased in the renal tissues of group $B$, this finding was also reported by Zafar et al., 2009 and they explained this finding by cellular damage in the kidney caused by STZ-induced diabetes $^{(6)}$. This explanation may be supported in this study by the significant decrease in ALT and ALP of group C with marked improvement in renal pathology and a lesser degree of tissue damage in this group. At the same time AST level in kidney of animals of group C was not decreased, so cell damage is not the only explanation of increased enzymes in kidney of diabetic animals and other mechanisms may be shared in this finding. Ramesh and Pugalendi 2006 attributed the increased ALT level in diabetes to insulin deficiency as Insulin suppresses the genes encoding gluconeogenic enzymes and ALT is one of them ${ }^{(17)}$. ALP is present in all tissues of the body, especially in the cell membrane and the levels are high in the liver, kidney, bone and placenta ${ }^{(17)}$. Diabetes and hyper- lipidemia also cause cell damage by altering the cell membrane architecture, which results in enhanced activities of ALP in diabetic rats ${ }^{(18)}$.In other studies the activities of ALP were increased, while the activities of AST and ALT were not altered in the kidneys of diabetic animals in which diabetes was induced by $\mathrm{STZ}^{(19-21)}$. This conflicting observation and limitation of available data require further research to precisely address the enzymatic changes in kidney of diabetic animals.

\section{Conclusion}

Human umbilical cord blood CD34+ cell transplantation in diabetic rats had been able to improve blood glucose level, partially ameliorate the renal damage in diabetic rats, and consequently improve renal functions, renal histology and renal enzyme levels alteration in the kidneys of diabetic animals.

\section{Acknowledgement}

The authors are grateful to all members of the Medical Education department, Faculty of Medicine, Suez Canal University for their cooperation.

Source(s) of support

Maastricht University, Center for International cooperation in Development of Education (MUNDO)

Conflicts of interest

No conflict of interest.

\section{References}

1. Fadini GP and Avogaro A. It is all in the Blood: The multifaceted contribution of circulating progenitor cells in diabetic complications. Exp Diabetes Res. 2012: ID:742976, 1-8.

2. Ezquer $M E$, Ezquer $\mathrm{FE}$, Arango-Rodríquez $M L$, Conget PA. MSC transplantation: A promising therapeutic strategy to manage 
the onset and progression of diabetic nephropathy. Biol Res. 2012;45(3):289-296.

3. Carvalho EN de, Carvalho NAS de, Ferreira LM. Experimental model of induction of diabetes mellitus in rats. Acta Cir. Bras. 2003;18: 60-64.

4. Zhou $\mathrm{H}$, Tian HM, Long $\mathrm{Y}$, Zhang $\mathrm{XX}$, Zhong L, Deng L, Chen XH, Li XQ. Mesenchymal stem cells transplantation mildly ameliorates experimental diabetic nephropathy in rats. Chin Med J (Engl). 2009;122(21):25732579.

5. Park JH, Park J, Hwang SH, Han Ha, Ha H. Delayed treatment with human umbilical cord blood-derived stem cells attenuates diabetic renal injury. Transplant Proc. 2012;44(4):1123-1126.

6. Zafar M, Naeem-UL-Hassan NS, Ahmed M, Kaimkhani ZA. Altered kidney morphology and enzymes in strepto-zotocin-induced diabetic rats. Int. J. Morphol. 2009;27(3):783790.

7. Reinders ME, Fibbe WE, Rabelink TJ. Multipotent mesenchymal stromal cell therapy in renal disease and kidney transplantation. Nephrol Dial Transplant. 2010;25(1):17-24

8. Monti M, Perotti C, Del FC, Cervio M, Redi CA. Stem cells: Sources and therapies. Biol Res. 2012;45(3):207-214.

9. Masoad RE, Ewais MM, Tawfik MK, Abd ElAll HS. Effect of mononuclear cells versus pioglitazone on streptozotocin-induced diabetic nephropathy in rats. Pharmacol Rep. 2012;64(5):1223-1233.

10. Abdouh M, Talbot S, Couture R, Hassessian HM. Retinal plasma extravasation in streptozotocin-diabetic rats mediated by kinin $B 1$ and $B 2$ receptors. Br J Pharmacol 2008;154:136-143.

11. Hasein MA, Attia FM, Awad MM, Abdelaal $\mathrm{HA}$ and Elbarabary M. Effect of human umbilical cord blood CD34+ progenitor cells transplantation in diabetic mice. Int J Diabetes Dev Ctries. 2011;31(2):113-117.

12. Karthik D, Ravikumar S. A Study on the Protective Effect of Cynodon dactylon Leaves Extract in Diabetic Rats. Biomed Environ Sc. 2011;24(2):190-199.
13. Lee RH, Seo MJ, Reger RL, Spees JL, Pulin AA, Olson SD, Prockop DJ. Multipotent stromal cells from human marrow home to and promote repair of pancreatic islets and renal glomeruli in diabetic NODISCID mice. Proc Natl Acad Sci U S A. 2006;103 (46):17438-17443.

14. Volarevic $\mathrm{V}$, Arsenijevic N, Lukic $M L$, Stojkovic M. Concise review: Mesen-chymal stem cell treatment of the complications of diabetes mellitus. Stem Cells. 2011;29(1):510.

15. Wang S, Li Y, Zhao J, Zhang J, Huang Y. Mesenchymal Stem Cells Ameliorate Podocyte Injury and Proteinuria in a Type 1 Diabetic Nephropathy Rat Model. Biol Blood Marrow Transplant. 2013; 19 (4): 538546.

16. Wang XX, Jiang T, Shen Y, Caldas Y, Miyazaki-Anzai S, Santamaria H, Urbanek C, Solis $\mathrm{N}$, Scherzer P, Lewis L, Gonzalez FJ, Adorini L, Pruzanski M, Kopp JB, Verlander JW, Levi $M$. Diabetic nephropathy is accelerated by Farn-esoid $X$ receptor deficiency and inhibited by Farnesoid $X$ receptor activation in a type 1 diabetes model. Diabetes. 2010;59(11):2916-2927.

17. Ramesh B, Pugalendi KV. Impact of umbelliferone (7-hydroxycoumarin) on hepatic marker enzymes in strepto-zotocin diabetic rats. Indian J Pharma-col 2006; 38:209-210.

18. Udayakumar $\mathrm{R}$, Kasthurirengan $\mathrm{S}$, Mariashibu T S, Rajesh M, Anbazhagan V R, Kim S C, Ganapathi A, Choi CW. Hypoglycaemic and hypolipidaemic effects of Withania somnifera root and leaf extracts on alloxan-induced diabetic rats. Int J Mol Sci. 2009;10(5): 2367-2382.

19. Prakasam A, Sethupathy S, Pugalendi KV. Influence of Casearia Esculenta root extract on protein metabolism and marker enzymes in streptozotocin-induced diabetic rats. Pol. J. Pharmacol. 2004;56 (5): 587593.

20. McAnuff-Harding MA, Omoruyi FO, Asemota $\mathrm{HN}$. Intestinal disaccharidases and some renal enzymes in strepto-zotocininduced diabetic rats fed sapogenin extract 
from bitter yam (Dioscorea polygonoides).

Life Sci. 2006;78(22):2595-2600.

21. Kaleem M, Medha P, Ahmed QU, Asif M , Bano B. Beneficial effects of Annona Squamosa extract in streptozotocininduced diabetic rats. Singapore Med J. 2008;49(10):800-804. 